\title{
Black hole X-ray transients: Mass accumulation in the disk - constraints for the viscosity
}

\author{
E. Meyer-Hofmeister ${ }^{1}$ and F. Meyer ${ }^{1}$ \\ Max-Planck-Institut für Astrophysik, Karl Schwarzschildstr. 1, 85740 Garching, Germany
}

Received 3 November 2000 / Accepted 9 March 2001

\begin{abstract}
The outburst cycles of black hole X-ray transients are now generally understood as caused by a thermal instability in the accretion disk, the same mechanism as in dwarf novae outbursts. During quiescence the accretion occurs via a cool disk in the outer region but changes to a coronal flow/ADAF in the inner region. The transition to the coronal flow is caused by evaporation of matter from the cool disk. This process is an important feature for the disk evolution. We point out that if the disk is depleted during the outburst, e.g. by irradiation, its evolution during quiescence is independent of the detailed outburst luminosity decline. The mass accumulation during quiescence has to meet several constraints as the accretion rate, the recurrence time and the total outburst energy. We present a critical discussion of different ways to model X-ray transient outburst cycles and compare with the requirements from observations. For the case of only little mass left over after the outburst the observations indicate a frictional parameter in the cool disk of order $\alpha_{\text {cold }}=0.05$, similar to that in dwarf nova disks during quiescence, with no need to resort to much lower $\alpha$ values of order 0.005 .
\end{abstract}

Key words. accretion disks - black hole physics - X-rays: stars - stars: individual: A0620-00, Nova Vul 1988, Nova Mus 1991, 4U 1630-472

\section{Introduction}

Soft X-ray transients (SXT), also known as X-ray novae, contain a black hole or neutron star primary and a lowmass companion star. We consider here the black hole sources. These systems are characterized by the high luminosity X-ray outburst after a long-lasting quiescence and are usually detected in X-rays. An optical counterpart is known for a number of systems. During quiescence the optical light is dominated by the secondary star allowing detailed photometry and spectroscopy to determine the system parameters of the binary. A0620-00 was the first low-mass X-ray binary proven in this manner to contain a black hole (McClintock \& Remillard 1986). For this well observed system at the distance of only about $1 \mathrm{kpc}$ two outbursts in optical light were observed, originally interpreted as classical nova outbursts, 1917 (Nova Mon 1917) and 1975 . For several systems only one outburst is known, their recurrence time is longer than 30 , possibly 50 years. See Tanaka \& Shibazaki (1996) and Tanaka (2000) for a comprehensive description of the observations of X-ray transients. Chen et al. (1997) collected and discussed the available data for properties of X-ray and optical light

Send offprint requests to: E. Meyer-Hofmeister, e-mail: emm@mpa-garching.mpg.de curves. Charles (1998) also reviewed the X-ray, optical, radio and IR observations of black hole X-ray binaries.

SXTs and dwarf novae are similar in the respect that mass is transferred from a Roche lobe-filling low mass secondary star to a compact primary, a neutron star or black hole in SXTs, a white dwarf in the dwarf nova case. Accretion occurs via a disk. The evolution of the accretion disk and the triggering of an outburst therefore were modeled in the same way as for the dwarf nova outbursts. In the framework of the "disk instability model" the outburst cycles are understood as a thermal relaxation oscillation between a hot ionized and a cool unionized disk (Meyer \& Meyer-Hofmeister 1981). Whether the disk is in the cool or the hot state at certain radii depends on the depends the mass flow rate in the disk and the amount of mass accumulated, and this on the viscosity. The size of the disk is important, irradiation can be an essential fact. The first investigations for SXTs were carried out by Huang \& Wheeler (1989) and Mineshige \& Wheeler (1989). Later detailed investigations (Cannizzo et al. 1995; Cannizzo 1998, review 1999, 2000) focussed on modeling the exponential decline of the outburst lightcurve.

For the evolution of the disk during quiescence one feature is important in SXTs: it is necessary to have a truncated inner disk. Otherwise the disk cannot be cool throughout. Even with a very low mass flow rate the disk 
would reach high temperatures in the inner region. This hot region and the further outward cool region cannot exist together in a quasi-stationary state. Instead cooling and heating fronts would move inward and outward in the disk and change the disk back and forth between the hot and the cool state (Meyer 1981). This truncation of the geometrically thin cool disk naturally results from evaporation of mass in the inner disk region into a hot corona (Meyer \& Meyer-Hofmeister 1994). For the region further in the concept of an advection-dominated accretion flow (ADAF) was developed as a mode of accretion which allows to explain the low luminosity of X-ray transients in quiescence as well as in AGN. The review paper by Narayan et al. (1998) gives a detailed description of the physics of ADAFs as well as the investigations of this mode of accretion by several authors. The application to galactic black hole X-ray binaries and galactic nuclei was very successful in modeling the observed spectra. Best studied systems are A0620-00, V404 Cyg (Narayan et al. 1996, 1997), Nova Muscae (Esin et al. 1997) and GRO J1655-40 (Hameury et al. 1997).

The ADAF in the inner disk region together with the geometrically thin disk further out provides a consistent picture for the accretion in SXTs. From observations for the quiescent state properties of the accretion disk can be derived. The "standard disk instability model" for dwarf nova outbursts allows to understand the triggering of the outbursts in SXTs after the long recurrence time. This has been proven in the application to A0620-00 (MeyerHofmeister \& Meyer 1999, hereafter MHM99), using the viscosity values typically found for dwarf novae. There it was assumed that the disk is practically empty after the outburst due to the strong irradiation of the disk which prevents the disk from changing back to the cool state and causes the long lasting outburst decline (King \& Ritter 1998). If this is the case the accumulation of mass in the disk is independent of the details of the outburst. In view of the difficulties which arise in modeling the observed outburst decline (see e.g. Cannizzo 2000) it is an advantage to separate the two phases of disk evolution.

Since the result of King \& Ritter (1998) has important consequences for our treatment we shortly repeat here their argumentation. They discuss the optical/X-ray flux ratios already found in earlier investigations and point out that the optical emission during the outburst of X-ray transients is far too high to result from intrinsic dissipation only. They argue that irradiation from the central source is important during the outburst. The irradiation then prevents the disk from returning to the cool state until central accretion is greatly reduced. This is consistent with the long-lasting outburst decline. This is a convincing picture and it seems difficult to explain the outburst duration without such a long-lasting accretion and the consequential essential reduction of disk mass. The analysis of Shabaz et al. (1998) gives further confirmation for this picture (see Sect. 3.3).

Menou et al. (2000) model the full outburst cycle of A620-00 in a way that about $90 \%$ of the mass in the disk remain there during the outburst. Evaporation was taken into account in a rough approximation (details will be discussed later). They conclude that the viscosity in quiescence has to be very low $\left(\alpha_{\text {cold }}=0.005\right)$ to reproduce the long recurrence times. In their investigation the effect of disk irradiation during the outburst is not included, also the possibility to model A0620-00 with standard viscosity values as in MHM99 was not noted apparently.

The aim of the present investigation is a critical comparison of the predictions of various theoretical models, including earlier results from Cannizzo (1998), with the available data for black hole transients.

In Sect. 2 we show examples of computed disk evolution for A0620-00. Evaporation in the inner region reduces the accumulation of mass and for low mass transfer rates the instability can only marginally be triggered. The evaporation of mass therefore is the important feature which governs the disk evolution during the long-lasting quiescence and needs to be taken into account for the evolutionary computations in a way, so that the physics is included. In Sect. 3 we discuss how far the models agree with the requirements from observation. In Sect. 4 we focus on the total amount of mass accumulated when the outburst occurs and compare with collected data for several black hole SXTs. In Sect. 5 we discuss consequences for the viscosity in the case of long recurrence time. Additional computations shed light on the situation in systems with short recurrence time where an essential part of the mass in the disk might be left over after the outburst.

\section{Disk evolution during quiescence - models for A0620-00}

\subsection{Computational method}

Diffusion governs the evolution of the mass distribution in the disk. Given a black hole mass and an orbital period the mass transfer rate determines the disk evolution (given an initial mass distribution). For systems with long recurrence time we assume that the disk becomes depleted during the long-lasting outburst, caused by the irradiation which prevents the disk to go back to the cool state (King \& Ritter 1998). This has an essential consequence for the conclusions.

Our computer code includes the variation of the outer and inner disk edge during the evolution. The disk size changes as determined by redistribution of matter and angular momentum. The size is limited by the $3: 1$ resonance radius (Whitehurst 1988; Lubow 1991), which lies inside the tidal truncation radius for systems with a small mass ratio as the SXTs. For the initial size of the disk we assume $90 \%$ of the resonance radius. The maximal disk size is related to the orbital period and the secondary star mass. We take the secondary star mass according to the mass of a Roche-lobe filling main-sequence star for the given period. The location of the inner disk edge also varies. It is determined by the evaporation of matter into the corona. The total evaporation efficiency computed in a 
one-zone model is applied to this interval. For mass flow rates lower than about $0.01 \dot{M}_{\text {Edd }}\left(\dot{M}_{\text {Edd }}=40 \pi G M / \kappa c\right.$, $\kappa$ electron scattering opacity) and a coronal $\alpha$-parameter of 0.3 the evaporation rate (in $\mathrm{g} / \mathrm{s}$ ) can be approximated by the following formula

$\dot{M}_{\text {evap }}(M, r)=10^{14.9} \cdot\left(M / M_{\odot}\right)^{2.3} \cdot\left(r / 10^{9.5}\right)^{-1.2}$

with $M$ the mass of the compact star (see Liu et al. 1995; Meyer et al. 2000). Coronal friction like that of a standard disk leads to redistribution of angular momentum and also to a partial outward flow that is taken into account (Liu et al. 1997, application to evaporation around a white dwarf). A detailed description of the computational method is given in MHM99. The black hole mass an important parameter for the disk evolution, unfortunately can be deduced from the observations only within some range of uncertainty. We choose the rate of mass transferred from the secondary star to meet the requirements from observations. The initial surface density was assumed to be very low, of order of the critical surface density $\Sigma_{\mathrm{A}}$ (viscosity parameter 0.3 ) or even lower. Without irradiation the surface density remaining after an outburst usually is between the two critical surface densities $\Sigma_{\mathrm{A}}$ and $\Sigma_{\mathrm{B}}$. The specific choice does not matter for the outcome of the simulation, due to the accumulation of much more mass during the long quiescence until the outburst is triggered.

\subsection{Requirements from observations}

Given the orbital period (known from observations) and a black hole mass there are only two free parameters left, the rate of mass transfer from the companion star and the viscosity parameter in the cool disk $\alpha_{\text {cold }}$. But several requirements have to be fulfilled connected with quiescence and outburst (this is the same situation for all X-ray transients). The relevant data with which the results of modeling should agree are:

(1) The accretion rate in quiescence derived from ADAF model based spectral fits (Narayan et al. 1998);

(2) The radius of the transition from the outer geometrically thin disk to the inner ADAF region derived from the Kepler velocity which corresponds to the maximum velocity width of the accretion disk $\mathrm{H} \alpha$ emission line (e.g. see Marsh et al. (1994) and Orosz et al. (1994) for A0620-00);

(3) The amount of matter accreted during the outburst estimated from the outburst light curve (for an assumed distance);

(4) The outburst recurrence time.

The mass transfer rate affects the outcome for all these requirements. In the accretion disk the transition to an coronal flow occurs where the evaporation rate exceeds the mass flow rate in the disk. The computed evolution then self-consistently determines the location at each time. The transition radius becomes smaller with proceeding mass accumulation and hence increasing mass flow rate in the inner disk (compare Fig. 3, MHM99). The viscosity value affects the amount of mass in the disk, higher for lower viscosity.

\subsection{Matter accumulation}

For different models, all designed to describe the X-ray nova A0620-00, we show in Fig. 1 how the mass in the disk accumulates with time. The amount is highest in the model of Cannizzo (1998, Fig. 9, model with long recurrence time). The viscosity is chosen as proportional to $(h / r)^{1.5}, h$ pressure scale height. Less than $10 \%$ of the mass in the disk is accreted during the outburst. This is the scheme used successfully in modeling of dwarf nova outbursts, but probably not adequate if irradiation affects the mass flow during outburst. In recent computations of only the outburst (Cannizzo 2000) irradiation is included and much more mass accretes. In the modeling of Menou et al. (2000) $\alpha_{\text {cold }}=0.005$ is taken, irradiation is not included and most of the mass remains in the disk during the outburst. (In their investigation the disk evolution was computed with and without a truncated disk. But for the non-truncated disk and their further parameters chosen for modeling A0620-00 the recurrence time is only 5 years, see Fig. 1. We compare with their result for a truncated disk, Fig. 7.) The dashed line gives the total amount of mass transferred (in Cannizzo's model the total amount of mass transferred is practically the same as the amount accumulated).

The two lower curves in Fig. 1 (solid lines) show the mass accumulation in our models for 4 and $6 M_{\odot}$ black holes (viscosity in the cool disk $\alpha_{\text {cold }}=0.05$ ). The mass transfer rates were chosen to meet the constraints from observations (compare Table 1 in MHM 1999), $1.9 \times 10^{-10} M_{\odot} / \mathrm{yr}$ and $3.4 \times 10^{-10} M_{\odot} / \mathrm{yr}$, respectively. The higher amount for the disk around the $6 M_{\odot}$ black hole results from the then higher evaporation rate (see Eq. (1)). This has the consequence that the critical surface density can only be reached at larger distance $r$ and more mass needs to be stored in the disk before an outburst occurs (see Fig. 5, MHM99). The dashed line indicates the total amount of matter transferred for $4 M_{\odot}$. During the late quiescence an increasing part of the matter transferred is evaporated and accreted, less and less mass is still accumulating until the critical surface density for triggering the outburst is reached.

For comparison we show the disk evolution for a mass transfer rate only $5 \%$ lower (dot-dashed line - note that this is not a fluctuation but a difference of the average over several decades): no outburst occurs, the disk becomes stationary. Such a system resembles a case of faint nontransient X-ray binaries. As discussed in earlier work (Meyer-Hofmeister \& Meyer 2000) the consideration of such a stationary disk raises the question how many black hole binaries exist in permanent quiescent state (see also Menou et al. 1999). Certainly there is a range of transfer rates around the marginal rate where fluctuations could 
bring the system to the other class. Thus the division into the two classes, stable/unstable, is not sharp. But we do not expect this to be a model for the SXT outbursts as suggested with the "globally stable disks" where an essential part of all mass accumulated is transferred in a rare mass transfer fluctuation, discussed below.

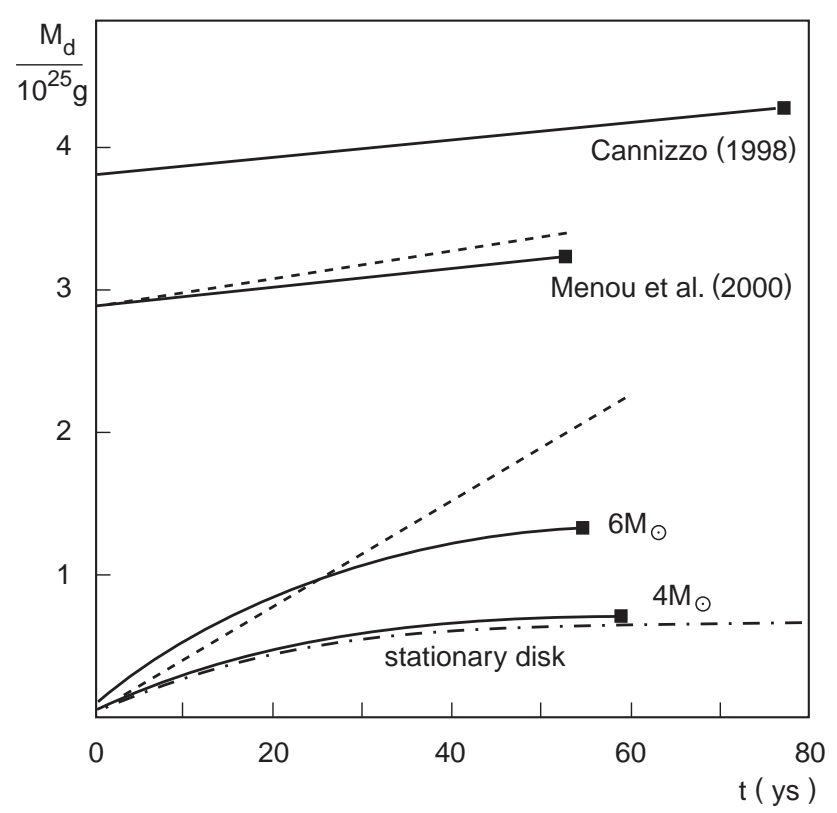

Fig. 1. Accumulation of mass in the disk during quiescence for different models, solid lines: Cannizzo (1998), Menou et al. (2000), Meyer-Hofmeister \& Meyer (1999), 4 and $6 M_{\odot}$, squares mark the onset of the outburst; dashed lines: total amount of mass transferred from the companion star; dasheddotted line: quasi-stationary disk, see text.

Menou et al. (2000) also considered a model of a "globally stable truncated disk", that means a disk where the surface density remains below the critical value for triggering an outburst. For this the mass transfer rate $10^{15} \mathrm{~g} / \mathrm{s}$ was taken, one third of the rate used for their "truncated disk instability model" where the outburst is triggered when the critical surface density is reached. In the globally stable disk model the outburst is thought to occur due to an increase of the rate of mass transfer from the secondary star. This means that an essential part of the mass needed for the onset of the outburst is brought over during the assumed phase of higher mass transfer. The authors mention that in such a case the finally accumulated mass in the disk and the resulting outburst is similar to that in the truncated disk instability model with a continuous accumulation of mass (compared before).

\subsection{Mass transfer variations}

Since an increase of the mass transfer rate is sometimes appealed to in connection with triggering an accretion disk instability we want to discuss here the timescales involved.

We note that what the recurrence time measures is the average of the mass transfer rate over the long time of quiescence, e.g. 50 years. This is a long time scale for atmospheres of late type companion stars. Thermal timescales of the outer layer relevant for mass transfer and other oscillation and relaxation times are much shorter. Variations of the mass transfer rate that might occur on such short timescales are thus averaged out and do not affect the cycle length. Long-term fluctuations of the transfer rate, on the other hand, are not achieved easily. It is a misconception that a fluctuation or change of the braking rate (e.g. by magnetized wind) results immediately in corresponding changes of the mass transfer rate as the secondary star otherwise will over- or under fill its Roche lobe. In order to change the mass transfer rate by a significant factor one must change the distance between stellar surface and inner Lagrangian point by a value of the order of an atmospheric scale height. This is typically of order $10^{-4}$ of the stellar radius. With orbital evolution timescales of $10^{9}$ years this gives $10^{5}$ years before any change of braking leads to the response of the mass transfer rate. Braking rate fluctuations on shorter timescales are smoothed out. For a discussion of this see Ritter (1988).

Observational evidence for a very stable transfer rate is present in the case of WZ Sge, a dwarf nova system with very long recurrence time, transferring mass via Roche lobe overflow. That system shows a repetitive cycle that is observed twice with nearly exactly the same cycle length of 32.6 and 32.4 years.

How well do the various models for A0620-00 now meet the requirements from observations?

\section{Comparison of models for A0620-00 with the observation}

\subsection{Observational requirement (1), evaporation rate - mass accretion rate}

The accretion rate found from the ADAF model $(M=$ $\left.6.1 M_{\odot}\right)$ fit of the spectrum of A0620-00 is $3-6 \times 10^{15} \mathrm{~g} / \mathrm{s}$ (Narayan et al. 1997, 1998). In Table 1 we summarize the results of the models with respect to the evaporation rate. To compare with the observations we consider the theoretical values for 20 years after the outburst that occurred 1975 , reduced by the wind loss $(\approx 20 \%$, Meyer et al. 2000). The theoretical evaporation rate should roughly agree with the accretion rate derived from observations. In Cannizzo's model the assumed evaporation gives values as low as $2 \times 10^{14} \mathrm{~g} / \mathrm{s}$. In the model of Menou et al. (2000) an assumed evaporation rate is taken, designed to be significantly smaller than the mass transfer rate and a parameter value $\varepsilon$ is chosen to ensure that the maximally truncated disk is still unstable. The value of the accretion rate after 20 years is $9 \times 10^{14} \mathrm{~g} / \mathrm{s}$, less than one third of the value derived from the observations. In our models the evaporation rate (Eq. (1)) is determined according to the evaporation theory and is not free. Only the parameter for the viscosity in the hot corona enters, chosen as 0.3 in all our computations (comparable to the parameter in the 
Table 1. Mass accretion rates for A0620-00.

\begin{tabular}{|c|c|c|c|}
\hline & $\begin{array}{r}\mathrm{BH} \text { mass } \\
\left(M_{\odot}\right) \\
\end{array}$ & $\alpha_{\text {cold }}$ & $\begin{array}{l}\dot{M}_{\text {acc }} \\
(\mathrm{g} / \mathrm{s}) \\
\end{array}$ \\
\hline $\begin{array}{l}\text { observations: } \\
\text { spectral fit by Narayan } \\
\text { et al. (1997, 1998) } \\
\text { (data McClintock } \\
\text { et al. 1995) }\end{array}$ & 6.1 & & $3-6 \times 10^{15}$ \\
\hline $\begin{array}{l}\text { models: } \\
\text { Cannizzo (1998) } \\
\text { Menou et al. (2000) } \\
\text { MHM99 } \\
\text { MHM99 }\end{array}$ & $\begin{array}{r}10 . \\
6 . \\
4 . \\
6 .\end{array}$ & $\begin{array}{l}\sim(h / r)^{1.5} \\
0.005 \\
0.05 \\
0.05\end{array}$ & $\begin{array}{r}2 . \times 10^{14} \\
9 . \times 10^{14} \\
3.7 \times 10^{15} \\
5.5 \times 10^{15}\end{array}$ \\
\hline $\begin{array}{l}\text { stationary disk } \\
\text { (MHM99) }\end{array}$ & 4. & 0.05 & $2.8 \times 10^{15}$ \\
\hline
\end{tabular}

Note: Model mass accretion rates taken 20 years into quiescence, which corresponds after the outburst in 1975 to about the year 1995; for the models MHM99 the rates of accretion onto the black hole (evaporation rate minus $20 \%$ wind loss) are given.

ADAF modeling and to results of numerical simulations of MHD turbulence (Hawley et al. 1995).

\subsection{Observational requirement (2), transition radius}

For Cannizzo's model no results are given for the inner truncation of the disk. In the model of Menou et al. the transition radius follows from their choice of an evaporation formula and is about $10^{10} \mathrm{~cm} 20$ years after outburst. In our treatment of evaporation the transition radius depends on the mass flow rate in the disk, the location therefore changes during the evolution. For 20 years after an outburst the radii found from our computations for 4 and $6 M_{\odot}$ are close to $10^{10} \mathrm{~cm}$, in good agreement with observations. The inner disk edge derived from the maximum velocity of the $\mathrm{H} \alpha$ emission line, $v_{\text {in }}=2100 \mathrm{~km}$ (Orosz et al. 1994), is located at 1.3 or $1.1 \times 10^{10} \mathrm{~cm}$ for a 4.4 or $6.1 M_{\odot}$ black hole (Narayan et al. 1996, 1997).

\subsection{Observational requirement (3), total amount of mass in the disk}

Figure 2 shows the amount of mass $M_{\mathrm{d}}$ (total amount of mass in the cool disk) from model computations for A0620-00, together with data from observations. The computations for $4 M_{\odot}$ agree better with the total amount of mass derived from observations. In earlier investigations (Narayan et al. 1996) a value of $4.4 M_{\odot}$, corresponding to an inclination of $70^{\circ}$, was used to model A0620-00, later Barret et al. (1996) suggested an inclination of $55^{\circ}$ which leads to a black hole mass of $6.1 M_{\odot}$. The evaporation efficiency still depends on the exact value of the viscosity used for the hot coronal gas. The result might change somewhat if another value would be chosen, further investigations are planned. The results of Cannizzo (1998) and Menou et al. (2000) are based on model computations where during the outburst most of the mass remains in the disk. In Fig. 2 we therefore also give the amount $\Delta M_{\mathrm{d}}$ accreted during the outburst. If irradiation during the outburst were not important the value $\Delta M_{\mathrm{d}}$ of Menou et al. (2000) would be in agreement with the estimates from observations. The key assumption of an empty disk after the outburst, used in our models, leads to a much lower amount of mass. (In the recent investigation of Cannizzo (2000) irradiation during the outburst is included and more mass than in the foregoing model is accreted; but the disk evolution in quiescence is not computed). In the next section we compare more general results for disks of different size with observations.

\subsection{Observational requirement (4), recurrence time}

The models of Cannizzo (1998) and Menou et al. (2000) were developed to show that long recurrence times can be achieved. Slight changes in the parameters choosen might reproduce the exact recurrence time, certainly involving also changes in the mass accumulation. Our computations yield the observed recurrence time together with all the other requirements from observations.

\section{Mass accumulation in disks around black holes of different mass}

\subsection{SXTs and WZ Sge stars}

A similarity of SXTs and WZ Sge stars, a subgroup of dwarf novae, was often pointed out (see e.g. Kuulkers 1999). This similarity concerns the long recurrence times and the long duration of the outbursts, the best example being WZ Sge (Patterson et al. 1981). For a review on the modeling of WZ Sge stars see Osaki (1995). The total amount of mass accreted on to the white dwarf during an outburst $\left(1-2 \times 10^{24} \mathrm{~g}\right.$, Smak 1993) is actually of the same order of magnitude as the amount of mass involved in SXT outbursts (Chen et al. 1997).

However the different disk size in these two classes of systems has interesting consequences for the viscosity (in Sect. 5.3 we discuss its possible origin). The size of the disk in WZ Sge (orbital period $81.3 \mathrm{~min}$ ) is only $1.6 \times 10^{10} \mathrm{~cm}$, limited by the $3: 1$ resonance radius. In A0620-00 the disk is roughly ten times larger. To store the same amount of mass as accumulated in SXT disks in the much smaller disk of WZ Sge requires a lower value of the viscosity parameter $\alpha_{\text {cold }}$ in WZ Sge systems $(0.001$, Meyer-Hofmeister et al. 1998). In the larger SXT disks, on the other hand, the similar amount of accumulated mass points to a standard value of a few times 0.01 . 
Table 2. X-ray transients established as black-hole sources.

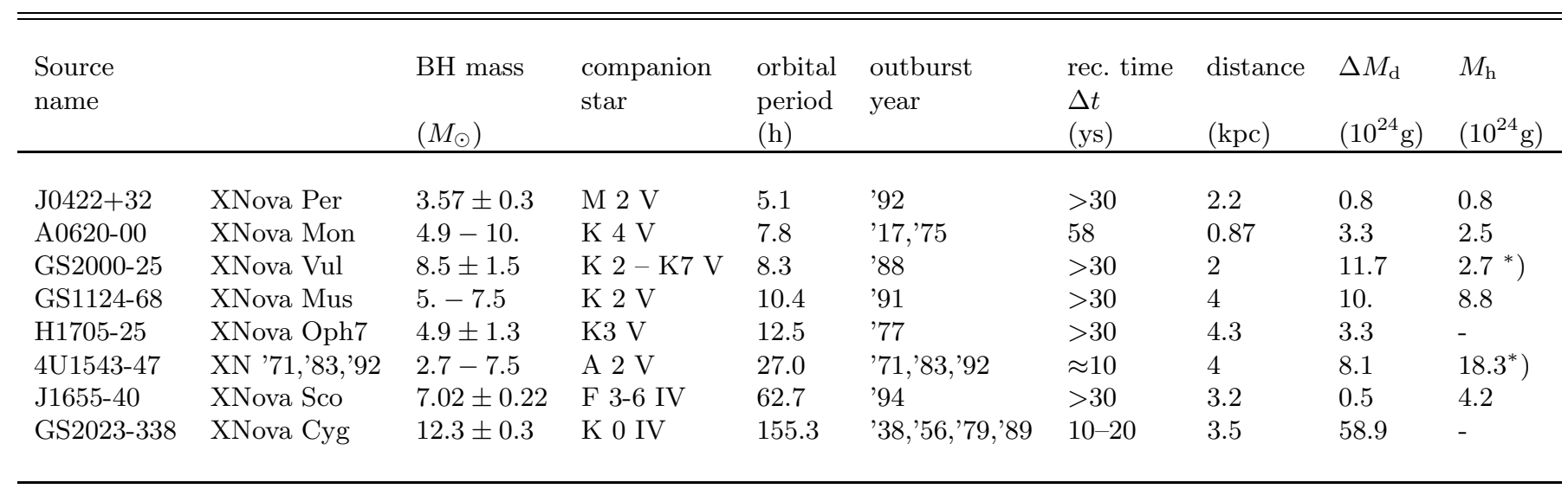

Note: Data for black hole mass, spectral type of companion star, orbital period, outburst year, distance and mass accreted during outburst $\Delta M_{\mathrm{d}}$ from Chen et al. (1997, see references therein); given in addition is $M_{\mathrm{h}}$, the amount of mass in the disk heated at the start of the outburst $M_{\mathrm{h}}$, estimated by Shabaz et al. (1998) (see text).

Data from other sources: distance for GS $1124-68$ as used by Shabaz et al. (1998), $\Delta M_{\mathrm{d}}$ according to this value; companion star spectral type for $4 \mathrm{U} 1543-47$ from Tanaka $\left.(2000) .{ }^{*}\right)$ the difference between $\Delta M_{\mathrm{d}}$ and $M_{\mathrm{h}}$ arises from a discrepancy in the value $\Delta M_{\mathrm{d}}$ for GS $2000+25$, and from the larger distance, $8 \mathrm{kpc}$, for $4 \mathrm{U} 1543-47$ (Shabaz et al. 1998) respectively.

\subsection{Data from observations of $S X T s$}

In Table 2 we give data for systems established as black hole transient sources. Knowledge of the distance of the sources is important for the estimate of the luminosity and therefore also the outburst energy and amount of accreted mass. Unfortunately for some systems, e.g. GS 1124-68, the values are very uncertain. For J0422+32 recently the distance $1.39 \mathrm{kpc}$ was derived (Webb et al. 2000). The properties of black hole transients compiled in the reviews of Tanaka \& Shabazaki (1996), Chen et al. (1997), Charles (1998) and Tanaka (2000) mainly differ with respect to the black hole mass estimates.

For our modeling the black hole masses are important, since the evaporation efficiency depends on it. Chen et al. (1997) derived estimates for the amount of mass accreted during the outburst from the outburst light curves of the systems. In the analysis of Shabaz et al. (1998) the exponential/linear decays of the light curves were studied and estimates for the amount of mass heated during the outburst were derived.

\subsection{Comparison with observations}

In Fig. 2 we show more general results for mass accumulation in disks around 4 and $6 M_{\odot}$ black holes. The disk size depends on the orbital period. We computed the disk evolution for periods from 4 to 12 hours and different mass transfer rates. The range of rates was taken as follows: the minimal rate arises from the fact that for too low rates no outburst occurs anymore (the higher the rate the more often outbursts are triggered), we took the maximal rate as the one which initiates outbursts every ten years. We did not want to consider shorter recurrence time cycles for our analysis of transients. The shaded areas in Fig. 2 mark the range of values of total mass accumulated corre- sponding to this range of transfer rates. Our two models for A0620-00 lie in these ranges. Later in this paper we compare with data for a number of SXTs observations.

In a very detailed investigation of outburst light curves of SXTs Chen et al. (1997) derived estimates for the amount of mass accreted. We show these values in Fig. 2 (for GS 1124-68 we have taken the smaller distance $4 \mathrm{kpc}$ as used in Shabaz et al. 1998). These values are uncertain if the distance is not well known. In their investigation of the effect of irradiation of the accretion disk during the outburst Shabaz et al. (1998) give estimates for the mass in the disk heated in the outburst. The question is whether this amount of mass includes all mass present in the disk at the time an outburst is triggered. The authors found that the radius of the hot disk at the peak of the outburst is comparable to the circularization radius. For A0620-00 they give the value $0.6 \times 10^{11} \mathrm{~cm}$. Therefore the main part is indeed hot. We give the values of heated mass in Fig. 2. All estimates for the amount of mass accreted in the outburst or heated are in the range $10^{24}$ to $1.2 \times 10^{25} \mathrm{~g}$.

\section{Viscosity in the cold disk}

\subsection{Systems with long recurrence time}

We first discuss the situation in systems with long recurrence time and long-lasting outbursts. If in these systems the irradiation causes the long-lasting decay (King \& Ritter 1998) the disk is depleted. The triggering of the outbursts after decades can well be modelled with a standard value around 0.05 as usual for dwarf nova outburst computations (all the observational requirements are fulfilled, see Sect. 3 and earlier work (MHM99)). Inspite of this result Menou et al. (2000) claimed that the viscosity would have to be low $(\approx 0.005)$ to model the SXT outbursts. Disk structure calculations show that the surface density 


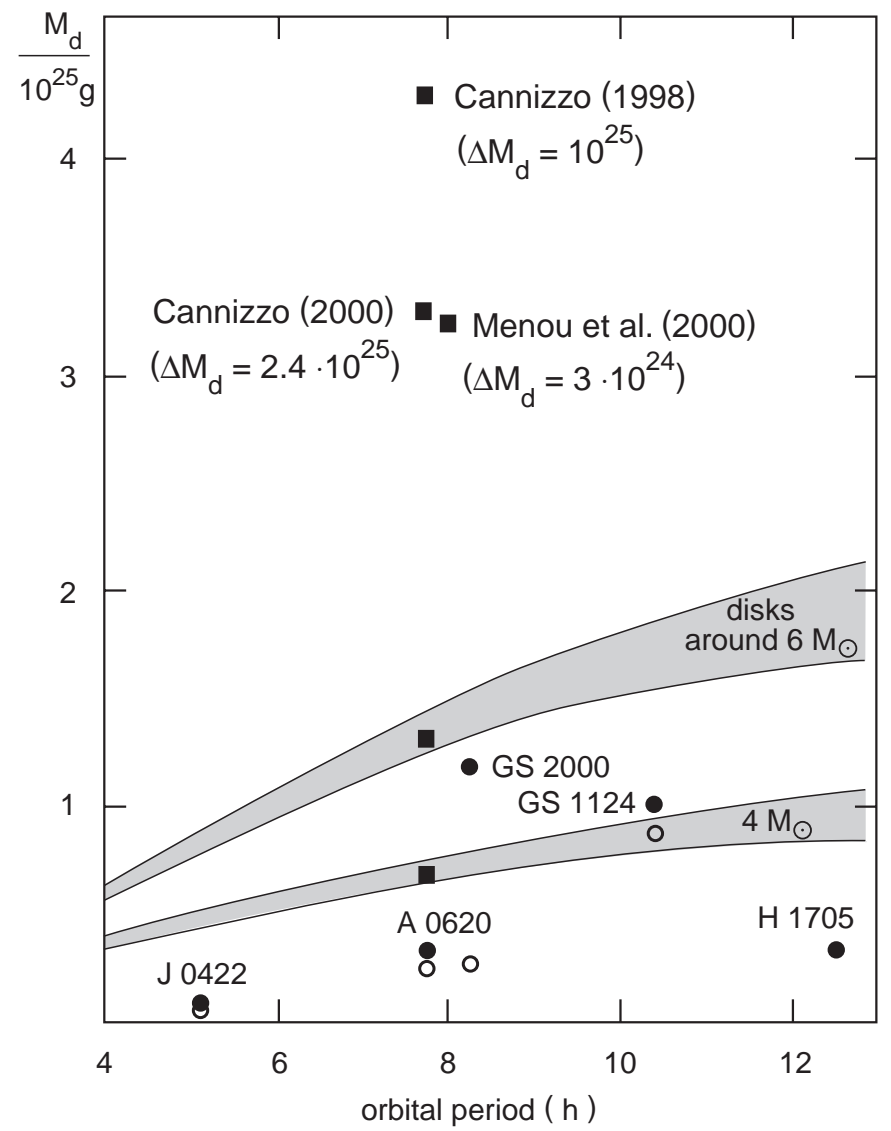

Fig. 2. Comparison of the total amount of mass accumulated in the disk for the outburst, in theoretical models and derived from observations. Models: shaded areas: results for disks of different size, for an appropriate range of mass transfer rates (discussed in the text). Observations: filled circles: values from observed outburst lightcurves (from Chen et al. 1997), open circles: estimates for the amount of heated mass in the disk (from Shabaz et al. 1998), compare Table 2.

and therefore the mass in the disk scales with $\alpha^{-0.8}$ approximately. For a viscosity ten times lower the amount of mass accumulated and the outburst energy would be roughly ten times higher. In the modeling with low viscosity the way out always is that only a small part of the total mass accretes. This is in conflict with the effect of irradiation and in general disagrees with the above mentioned amounts of mass heated up in the disk.

\subsection{Systems with short recurrence time}

There are a few systems with more frequent outbursts. $4 \mathrm{U}$ 1630-472 e.g. has outbursts about every two years. Their duration differs, also the observed luminosity which reaches values around $4 \times 10^{38} \mathrm{erg} / \mathrm{s}$. The amount of mass accumulated in the disk is derived as several $10^{25} \mathrm{~g}$, based on the uncertain distance of $10 \mathrm{kpc}$ (values from Chen et al. 1997). It is unclear whether the disk is empty after the outburst.
In an earlier investigation such short cycles were already computed assuming a depleted disk and $\alpha_{\text {cold }}=0.05$ (Meyer-Hofmeister \& Meyer 2000). Now we performed test computations to clarify whether, in principle, these short outburst cycles can also be modeled with a not depleted disk and the standard viscosity value $\alpha_{\text {cold }}=0.05$. The black hole mass and the orbital period of $4 \mathrm{U} 1630-472$ are not known. We performed test calculations for $6 \mathrm{M}_{\odot}$ and 7.75 hours (period as A0620-00). For the initial mass distribution we assume a surface density distribution as adequate from dwarf nova outburst modeling experience $\left(\log \Sigma_{\text {initial }}(r)=0.5\left(\log \Sigma_{\mathrm{A}}(r)+\log \Sigma_{\mathrm{B}}(r)\right), \Sigma_{\mathrm{A}}\right.$ and $\Sigma_{\mathrm{B}}$ critical surface density values), viscosity in the hot state $0.3)$. The mass transfer rate $2 \times 10^{-9} M_{\odot} / y$ is high enough to produce the outburst after two years. The accumulated amount of mass in the disk is $1.1 \times 10^{25} \mathrm{~g}$, in rough agreement with the observations. We conclude that values of order 0.05 are also suitable to describe the short outbursts in cases where the disks are not depleted.

\subsection{The general picture of the origin of the viscosity in cold SXT disks during quiescence}

The values, $\alpha_{\text {cold }} \approx 0.05$, in quiescent X-ray transients appear to support a general picture of magnetic friction in disks in SXTs and dwarf novae. In outburst the magnetic field caused viscosity (Balbus \& Hawley 1991, 1992) is high sustained by dynamo action in the accretion disk (for recent numerical simulations see Hawley et al. 1995; Brandenburg et al. 1995; Armitage 1998; Hawley 2000). As the disk returns to the quiescent state and becomes cool the electrical conductivity drops and the dynamo can no longer operate (Gammie \& Menou 1998), the high outburst viscosity vanishes. However a magnetosphere of the secondary star in which the accretion disk is embedded can still provide magnetic flux through the disk on which the Balbus-Hawley mechanism can work and supply a smaller magnetic friction in quiescence than that in outburst. WZ Sagittae systems have probably lost this source of magnetic flux since their secondaries have become cool degenerate brown dwarfs which can not sustain dynamo action or keep fossil fields in their interior. This could explain why their remaining viscosity is so much smaller than that of all other systems in quiescence though they have similar viscosity in outburst (Meyer \& Meyer-Hofmeister 1999). Then the derived $\alpha$-values for SXTs in quiescence would indicate that their secondaries have magnetospheres. In this respect we also note that these stars appear to be evolved (King et al. 1996) and tend to have outer convective zones, an ingredient for solar type dynamos.

\section{Conclusions}

King \& Ritter (1998) argued that strong irradiation during the long-lasting outbursts of SXTs keeps the disk hot, and almost all mass in the disk flows towards the black hole. We make the key assumption that in black hole transients with long recurrence time the disk is significantly 
depleted at the end of the outburst. The estimates for the amount of mass in the disk heated during the outburst (Shabaz et al. 1998) confirm this picture. When one follows the disk evolution and mass accumulation one finds that the long recurrence times can well be modelled with a "standard" viscosity value $\alpha_{\text {cold }}=0.05$. Evaporation of the inner disk region is an important feature in such disk evolution. All constraints from observation are fulfilled. For a full outburst cycle, where the disk evolution during quiescence computed here would be completed with the computation of the run of the outburst follows: the amount of matter accumulated agrees with the estimates from the outburst lightcurve. Only the features of the detailed lightcurve are not determined, those depending on parameters as the viscosity in the hot state and irradiation. We point out that the evaporation efficiency is taken from a physical model (Meyer \& Meyer-Hofmeister 1994) and no ad hoc parameter fitting was used. The computations of Menou et al. (2000) are based on a simulation of evaporation with free parameters which ensure that the evaporation rate is significantly below the mass transfer rate and, more severe, the maximally truncated disk is still unstable. Such a strategy does not allow a discrimination between stable and unstable models.

The models of Cannizzo (1998, $\alpha$ proportional to $(h / r)^{1.5}, h$ pressure scaleheight) and Menou et al. (2000, $\left.\alpha_{\text {cold }}=0.005\right)$, suggest that only a small part of the total mass in the disk is accreted during an outburst. But, even if the disk in A0620-00 would not be depleted the models only agree marginally with the other requirements from observations.

We further find that SXTs with a recurrence time as short as two years can also be modeled with $\alpha_{\text {cold }}=0.05$, assuming a depleted or a not depleted disk.

We thus conclude that the accretion disk in SXTs in quiescence display rather standard cold disk friction common to SXTs and dwarf novae and do not need nor indicate very low $\alpha$-values of order 0.005 .

\section{References}

Armitage, P. J. 1998, ApJ, 501, L189

Balbus, S. A., \& Hawley, J. F. 1991, ApJ, 376, 214

Balbus, S. A., \& Hawley, J. F. 1992, ApJ, 400, 610

Barret, D., McClintock, J. E., \& Grindlay, J. E. 1996, ApJ, 473, 963

Brandenburg, A., Nordlund, A., Stein, R. F., et al. 1995, ApJ, 446,741

Cannizzo, J. K. 1998, ApJ, 494, 366

Cannizzo, J. K., 1999, in Proc. of Disk Instablities in Close Binary Systems, ed. S. Mineshige, \& J. C. Wheeler (Universal Academic Press, Kyoto), 177

Cannizzo, J. K. 2000, ApJ, 534, L35

Cannizzo, J. K., Chen, W., \& Livio, M. 1995, ApJ, 454, 880

Charles, P. 1998, in The Theory of Black Hole Accretion Discs, ed. M. A. Abramowicz, G. Bjornsson, \& J. E. Pringle (Cambridge University Press), 1

Chen, W., Shrader, C. R., \& Livio, M. 1997, ApJ, 491, 312

Esin, A. A., McClintock, J. E., \& Narayan, R. 1997, ApJ, 489, 865
Gammie, C. F., \& Menou, K. 1998, ApJL, 492, L75

Hameury, J.-M., Lasota, J.-P., McClintock, et al. 1997, ApJ, 489,234

Hawley, J. F. 2000, ApJ, 528, 462

Hawley, J. F., Gammie, C. V., \& Balbus, S. A. 1995, ApJ, 440, 742

Huang, M., \& Wheeler, J. C. 1989, ApJ, 343, 229

King, A. R., \& Ritter, H. 1998, MNRAS, 293,L42

King, A. R., Kolb, U., \& Burderi, L. 1996, ApJ, 464, L127

Kuulkers, E. 1999, in Proc. of Disk Instabilities in Close Binary Systems, ed. S. Mineshige, \& J. C. Wheeler (Universal Academic Press, Kyoto), 169

Liu, F. K., Meyer, F., \& Meyer-Hofmeister, E. 1995, A\&A, 300, 823

Liu, F. K., Meyer, F., \& Meyer-Hofmeister, E. 1997, A\&A, 328, 247

Lubow, S. H. 1991, ApJ, 381, 268

Marsh, T. R., Robinson, E. J., \& Wood, J. H. 1994, MNRAS, 266,137

McClintock, J. E., \& Remillard, R. A. 1986, ApJ, 308, 110

McClintock, J. E., Horne, K., \& Remillard, R. A. 1995, ApJ, 442358

Menou, K. 2000 [astro-ph/0009022]

Menou, K., Narayan, R., \& Lasota, J.-P. 1999, ApJ, 513, 811

Menou, K., Hameury, J.-M., Lasota, J.-P., \& Narayan, R. 2000, MNRAS, 314, 498

Meyer, F., \& Meyer-Hofmeister, E. 1981, A\&A, 104, L10

Meyer, F., \& Meyer-Hofmeister, E. 1999, A\&A, 341, L23

Meyer, F., \& Meyer-Hofmeister, E. 1994, A\&A, 288, 175

Meyer, F., Liu, B. F., \& Meyer-Hofmeister, E. 2000, A\&A, 354, L67

Meyer-Hofmeister, E., \& Meyer, F. 1999, A\&A, 348, 154

Meyer-Hofmeister, E., \& Meyer, F. 2000, A\&A, 355, 1073

Meyer-Hofmeister, E., Meyer, F., \& Liu, B. F. 1998, A\&A, 339, 507

Mineshige, S., \& Wheeler, J. C. 1989, ApJ, 343, 241

Narayan, R., McClintock, J. E., \& Yi, I. 1996, ApJ, 457, 82

Narayan, R., Barret, D., \& McClintock, J. E. 1997, ApJ, 482, 448

Narayan, R., Mahadevan, R., \& Quataert, E. 1998, in The Theory of Black Hole Accretion Discs, ed. M. A. Abramowicz, G. Bjornsson, \& J. E. Pringle (Cambridge University Press), 148

Orosz, J. A., Bailyn, C. D., Remillard, R. A., et al. 1994, ApJ, 436,848

Osaki, Y. 1995, PASJ, 47, 47

Patterson, P. J., Mc Graw, J. T., Coleman, L., et al. 1981, ApJ, 248,1067

Ritter, H. 1988, A\&A, 202, 93

Shabaz, T., Charles, P. A., \& King, A. R. 1998, MNRAS, 301, 382

Smak, J. 1993, Acta Astron., 43, 101

Spruit, H. 1989, in Theory of Accretion Disks, ed. F. Meyer et al. (Kluwer Academic Publishers, Dordrecht), 325

Tanaka, Y., \& Shibazaki, N. 1996, ARA\&A, 34, 607

Tanaka, Y. 2000, in Highly Energetic Physical Processes and Mechanisms for Emission from Astrophysical Plasmas, IAU Symp., vol. 195, ed. P. C. H. Martens, S. Tsuruta, \& M. A. Weber, 37

Verbunt, F., \& Zwaan, C. 1981, A\&A, 100, L7

Webb, N. A., Naylor, T., Ioannou, Z., et al. 2000, MNRAS, 317,528

Whitehurst, R. 1988, MNRAS, 232, 35 